\title{
Cardiac Involvement in a Patient With POEMS Syndrome Detected Using Cardiac Magnetic Resonance Imaging
}

\author{
Guoyuan PeI, ${ }^{1}$ BMSci, Dan YANG, ${ }^{1}$ BMSci, Jiayu Sun, ${ }^{2}$ MD, Yong Luo, ${ }^{1}$ BMSci, \\ Jiaqi YAN, ${ }^{2}$ BMSci, and Yucheng CHEN, ${ }^{1}$ MD
}

\begin{abstract}
SUMMARY
We present a case of POEMS syndrome in which cardiac MRI (CMR) revealed cardiac involvement. POEMS syndrome is a very rare disease and cardiac involvement in POEMS syndrome is rarely reported. In the present case, echocardiography revealed left ventricular hypertrophy with moderately impaired systolic function and moderate pulmonary hypertension. CMR examination showed a typical circular subendocardial type of late gadolinium enhancement, which is a classic pattern of cardiac amyloidosis. The diagnosis of cardiac amyloidosis in POEMS would be helpful for further risk stratification and treatment strategies. (Int Heart J 2015; 56: 571-573)
\end{abstract}

Key words: Delayed enhancement, Left ventricular hypertrophy, Cardiac amyloidosis

$\mathrm{P}$ OEMS syndrome is a very rare disease which is associated with underlying plasma cell disease, and it typically consists of polyradiculoneuropathy, organomegaly, endocrinopathy, monoclonal plasma cell disorder, and skin changes. The diagnosis of POEMS syndrome is based on comprehensive clinical and laboratory tests. The confirmed diagnosis criteria are met based on the existence of two mandatory major criteria including polyneuropathy, monoclonal plasma cell proliferation disease, one of the 3 other major criteria (Castleman disease, sclerotic bone lesions and VEGF elevation), and any one of 6 minor criteria (organomegaly, extravascular volume overload, endocrinopathy, skin changes, papilledema, and thrombocytosis/polycythemia). ${ }^{1)}$ Organomegaly is one of the common features of POEMS syndrome, with spleen, hepato or lymph nodes being the most affected organs. Cardiac involvement in POEMS has rarely been reported. Pulmonary hypertension and secondary right ventricle changes are considered as one component of POEMS syndrome. ${ }^{1)}$ In the cases of POEMS syndrome reported thus far, cardiac involvement was detected by echocardiography or endomyocardial biopsy, for which the majority of patients usually do not provide consent. In the present case, we discuss the role of CMR in detecting cardiac involvement in POEMS syndrome.

\section{Case Report}

A 61-year-old woman complained of persistent paresthesia of the fingers and lower limb edema for 1 year. She consulted a local hospital due to the uncomfortable symptom, but a definite diagnosis could not be made. The patient later experienced shortness of breath and orthopnea and was transferred to our institution. The patient has a past medical history of well controlled asthma. Physical examination revealed stable vital signs and normal skin. A mildly dilated jugular vein was seen. The lung fields were clear. Furthermore, the cardiac border was enlarged, but no murmur could be heard. The liver and spleen could not be palpated. However, there was remarkable pitting edema on both legs. A 12-lead electrocardiogram showed low-voltage areas in the limb leads (Figure 1). A thoracic CT scan showed pleural effusion, dilation of the pulmonary artery, cardiomegaly, and pericardial effusion (Figure 2). Abdominal ultrasound examination revealed seroperitoneum, but no hepatosplenomegaly. Electroneuromyography (EMG) demonstrated peripheral neurogenic disorder. Laboratory tests revealed hypothyroidism (TSH: $6.05 \mathrm{mU} / \mathrm{L}$, normal range: 0.27-4.2 mU/L. FT3: $3.02 \mathrm{pmol} / \mathrm{L}$, normal range: $3.60-7.50$ $\mathrm{pmol} / \mathrm{L}$; FT4: $10.23 \mathrm{pmol} / \mathrm{L}$, normal range: $12.0-22.0 \mathrm{pmol} / \mathrm{L}$ ). Immunofixation electrophoresis showed a monoclonal light chain $(\lambda)$ component, and $\mathrm{M}$ protein was detected by serum protein electrophoresis. Bone marrow biopsy showed $8 \%$ infiltration of monoclonal $(\lambda)$ plasma cells (Figure 3). Based on all these clinical findings, POEMS syndrome was diagnosed. The patient underwent further evaluation of the heart due to congestive heart failure. Echocardiography revealed left ventricular hypertrophy (LVH) with moderately impaired systolic function and moderate pulmonary hypertension (estimated systolic pulmonary artery pressure was $59 \mathrm{mmHg}$ ) (Figure 4). A comprehensive CMR examination was performed to detect possible cardiac involvement. CMR examination revealed moderately decreased global left ventricular global function and concentric LVH. Right ventricular global function was also moderately decreased. Myocardial delayed enhancement imaging showed a typical circular subendocardial type of late

From the ${ }^{1}$ Division of Cardiology and ${ }^{2}$ Department of Radiology and Department of Pathology, West China Hospital, Sichuan University, Chengdu, China.

Address for correspondence: Yucheng Chen, MD, Division of Cardiology, West China Hospital, Sichuan University, Chengdu, Sichuan Province 610041, China. E-mail: chenyucheng2003@126.com

Received for publication January 24, 2015. Revised and accepted March 13, 2015.

Released in advance online on J-STAGE September 4, 2015.

All rights reserved by the International Heart Journal Association. 


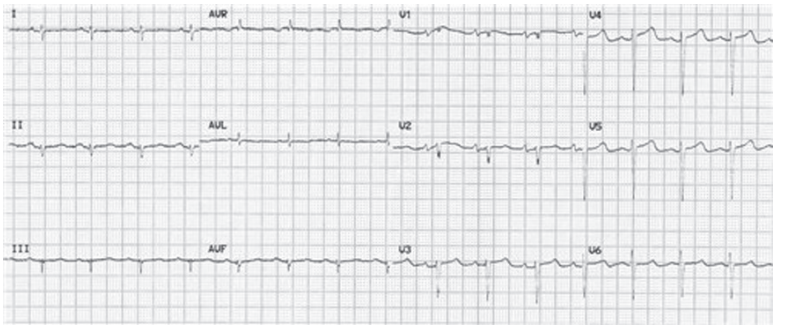

Figure 1. 12-lead electrocardiogram showed low-voltage areas in the limb leads.

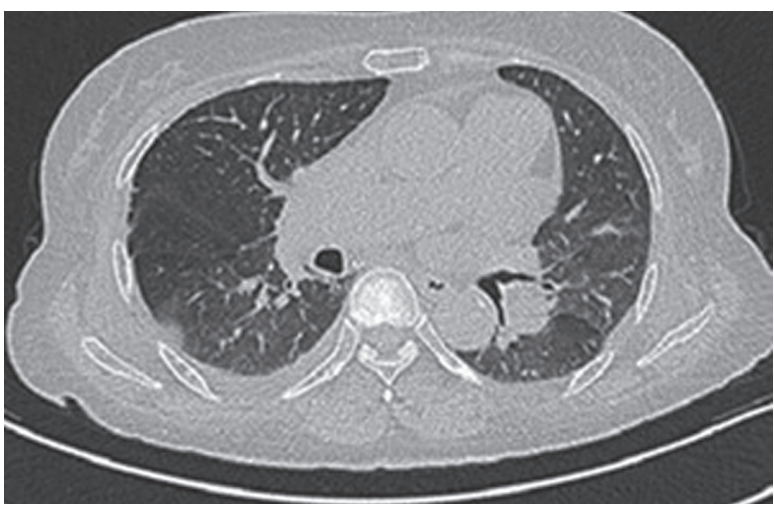

Figure 2. Thoracic CT scan showed pleural effusion, dilation of the pulmonary cardiomegaly, and pericardial effusion.

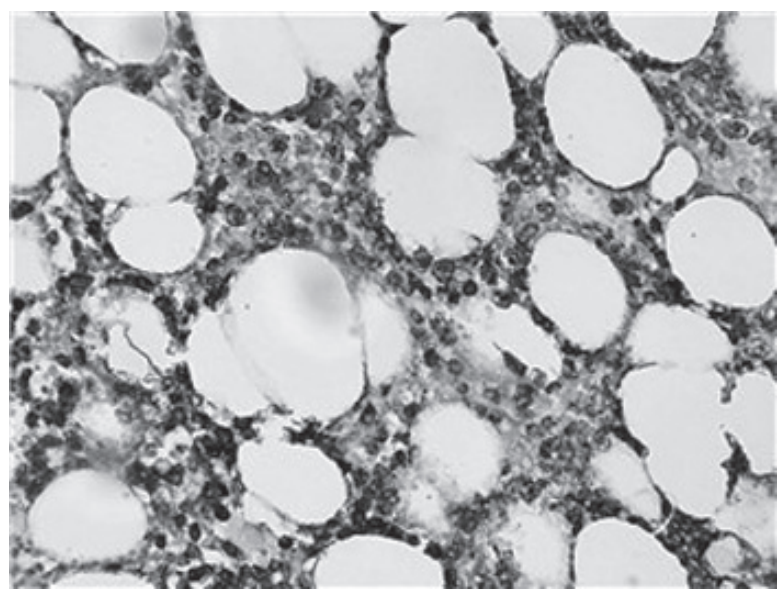

Figure 3. Immunohistochemistry of bone marrow biopsy showed infiltration of monoclonal $(\lambda)$ plasma cells

gadolinium enhancement, which is a classic pattern of cardiac amyloidosis (Figure 5). The patient received chemotherapy with bortezomib plus corticosteroid along with supportive care. After the treatment, the dyspnea gradually improved and the lower limb edema was alleviated.

\section{Discussion}

We have presented a case of POEMS syndrome in which
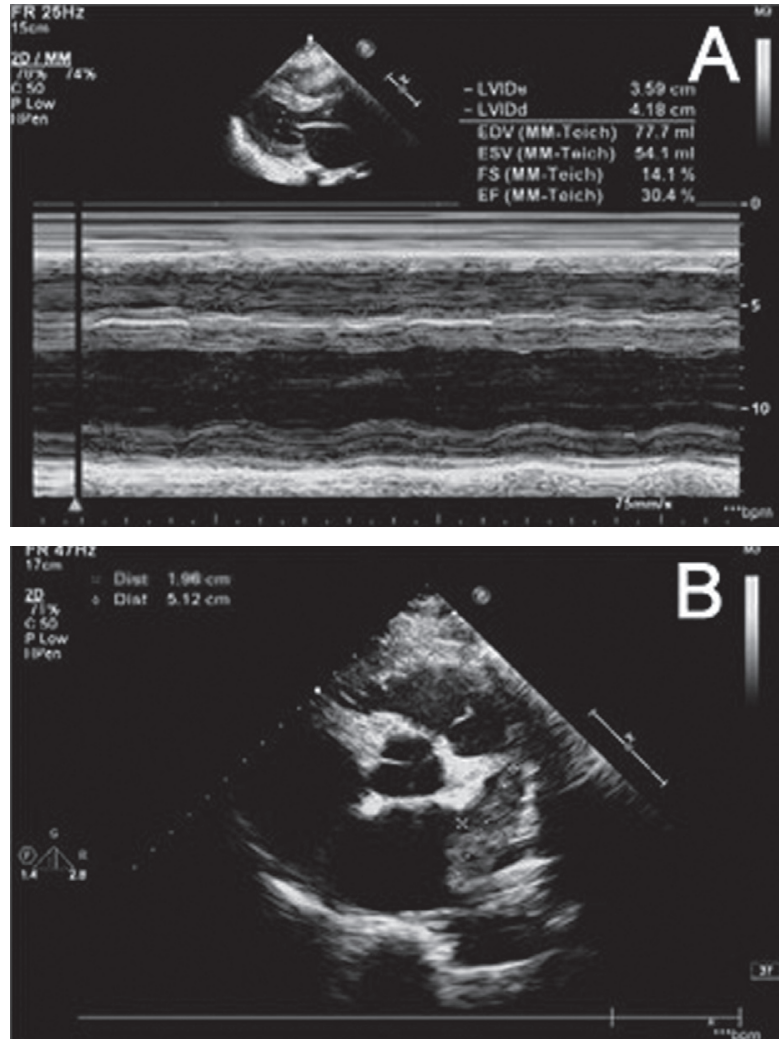

Figure 4. Echocardiography. A and $\mathbf{B}$ revealed left ventricular concentric hypertrophy (interventricular septal thickness, left ventricular posterior wall thickness, left ventricular end-diastolic diameter, and left ventricular end-systolic diameter were $15,12,42$, and $36 \mathrm{~mm}$, respectively) with moderately impaired systolic function (left ventricular ejection fraction was $30.4 \%$ ). Moderate pulmonary hypertension was also revealed (estimated systolic pulmonary artery pressure was $59 \mathrm{mmHg}$ ).

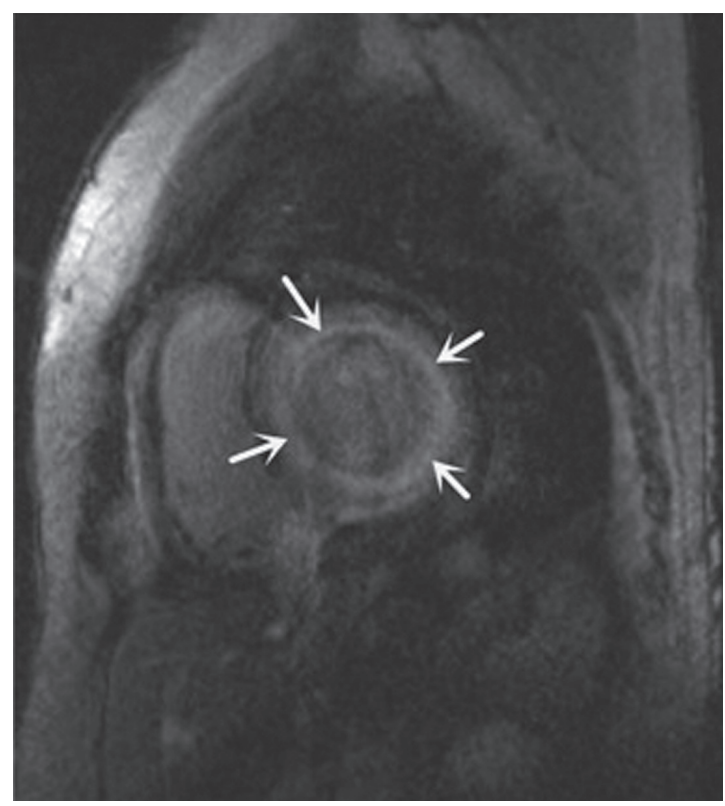

Figure 5. Myocardial delayed enhancement imaging of CMR showed the circular subendocardial type of late gadolinium enhancement. 
CMR demonstrated combined cardiac amyloidosis. The involvement of the left ventricle in POEMS has been overlooked in previous studies. The incidence and characteristics of left ventricle involvement are rarely reported. So far, heart failure and left ventricular hypertrophy in POEMS have been reported in only a few cases, and the underlying mechanism remains unclear. ${ }^{2)}$ The possible association between pulmonary hypertension and diastolic dysfunction of the left ventricle has been mentioned in one case report. ${ }^{3)}$ Perhaps this association should be emphasized to explore the mechanism of cardiac involvement in POEMS syndrome in future studies.

The coexistence of POEMS and cardiac amyloidosis confirmed by endomyocardial biopsy has been reported in only two cases up to now. ${ }^{4)}$ In the present case, myocardial delayed enhancement showed a typical late gadolinium enhanced (LGE) that was in accordance with cardiac amyloidosis. Based on the sensitivity and specificity of LGE in the diagnosis of cardiac amyloidosis, ${ }^{5)}$ the existence of cardiac amyloidosis or infiltration along with POEMS was highly possible in the present case. CMR was a good tool for the differential diagnosis of cardiomyopathy. Nureki, et al reported a case of cardiac sarcoidosis involving the lungs, eyes, and skin in which CMR showed T2-high intensity in the thickening of the interventricular septum which has abnormal accumulation of gallium-67. ${ }^{6}$ Soma, et al reported a case of ST-elevated myocardial infarction caused by amyloidosis deposition in intramural coronary arteries, and CMR showed that late gadolinium enhancement was widespread in the subendocardium. ${ }^{7)}$ Hosoya, et al reported a case of amniotic fluid embolism (AFE) with severe cardiopulmonary complications. Late gadolinium enhancement was detected at the mid-wall of the left ventricle which suggests the pathophysiological mechanism of severe cardiac complications in AFE. ${ }^{8)}$ The limitation of the this case was that the CMR was not reviewed because of the long chemotherapeutic time, long response time, and poor economic conditions.

POEMS syndrome is a paraneoplastic syndrome that generally has a good prognosis, whereas AL cardiac amyloido- sis has a poor outcome. Therefore, the diagnosis of cardiac amyloidosis in patients with POEMS will necessitate changes in risk stratification and treatment strategy. CMR was a good tool for the diagnosis of cardiac amyloidosis and should be used to explore the possible involvement in POEMS syndrome. To the best of our knowledge, this is the first case to report the coexistence of POEMS syndrome and cardiac amyloidosis which was diagnosed by CMR imaging. More cases and further studies on the application of CMR in POEMS syndrome will help to clarify the underlying cardiac involvement in this rare disease.

\section{REFERENCES}

1. Dispenzieri A. POEMS syndrome: 2014 update on diagnosis, riskstratification, and management. Am J Hematol 2014; 89: 214-23. (Review)

2. Tanus T, Miller HJ. POEMS syndrome presenting with cardiomegaly and cardiomyopathy. J Intern Med 1992; 231: 445-8.

3. Yokokawa T, Nakazato K, Kanno Y, et al. Pulmonary hypertension and refractory heart failure in a patient with Crow-Fukase (POEMS) syndrome. Intern Med 2013; 52: 1061-5.

4. Adami F, Briani C, Binotto G, Altinier S, Tarantini G, Semenzato G. Coexistence of primary AL amyloidosis and POEMS syndrome: efficacy of melphalan-dexamethasone and role of biochemical markers in monitoring the diseases course. Am J Hematol 2010; 85: 131-2.

5. Maceira AM, Joshi J, Prasad SK, et al. Cardiovascular magnetic resonance in cardiac amyloidosis. Circulation 2005; 111: 186-93.

6. Nureki S, Miyazaki E, Nishio S, et al. Interventricular septal thickening as an early manifestation of cardiac sarcoidosis. Int Heart J 2014; 55: 181-3

7. Soma K, Takizawa M, Uozumi H, et al. A case of ST-elevated myocardial infarction resulting from obstructive intramural coronary amyloidosis. Int Heart J 2010; 51: 134-6.

8. Hosoya Y, Watanabe M, Terashima M, et al. Cardiac magnetic resonance imaging in a patient with amniotic fluid embolism associated with severe cardiopulmonary complications. Int Heart J 2013; 54: 119-22. 